\title{
On the validity of Akasofu's $\varepsilon$ parameter and of the Vasyliunas et al. general formula for the rate of solar wind-magnetosphere energy input
}

\author{
Takao Aoki \\ Nagano Technical High School, Nagano 380-0948, Japan \\ (Received March 22, 2004; Revised December 3, 2004; Accepted January 11, 2005)
}

\begin{abstract}
The $\varepsilon$ parameter of Akasofu and the general formula of Vasyliunas et al. (1982) have been widely used as functions representing the rate of the solar wind energy input into the Earth's magnetosphere. These two functions have a feature that dependence on the interplanetary magnetic field (IMF) is assumed to be expressed by the combination of $B$, the magnitude of the IMF, and $\sin (\theta / 2)$, where $\theta$ is the clock angle, or by the combination of $B_{T}=\left(B y^{2}+B z^{2}\right)^{1 / 2}$, the magnetic field perpendicular to the Sun-Earth line, and $\sin (\theta / 2)$, not by the $B z$ component. We check the validity of this assumption by presuming that the $A L$ index has good correlation with the solar wind energy input rate and by applying the regression analysis in a power law form to hourly values of the $A L$ index and of solar wind parameters during the period 1966-1987. We show that $B z$ generally gives high correlation coefficients and shows higher consistency in exponents than the combination of $B$ (or $B_{T}$ ) and $\sin (\theta / 2)$. Thus we conclude that the IMF dependence of the rate of the solar wind energy input is described better by $B z$ than by the combination of $B$ (or $B_{T}$ ) and $\sin (\theta / 2)$.
\end{abstract}

Key words: Substorm, coupling function, $\varepsilon$ parameter, Vasyliunas et al. general formula, IMF clock angle.

\section{Introduction}

The magnetospheric substorm is a basic mode of the interaction between the solar wind and the Earth's magnetosphere. The function representing the rate of the solar wind energy input into the magnetosphere is called the energy coupling function between the solar wind and the magnetosphere, and its functional form has been investigated from both analytical and theoretical points of view over four decades (e.g., Baker, 1986; Koskinen and Tanskanen, 2002).

Simple functions proposed so far are $B z V$ (Rostoker et al., 1972), where $B z$ is the north-south component of the interplanetary magnetic field (IMF), and $V$ is the solar wind velocity, and $B s V^{2}$ (Murayama and Hakamada, $1975)$, where $B s$ is the southward component of the IMF: $B s=-B z$ for $B z<0$, and $B s=0$ for $B z \geq 0$. The above two expressions have a feature that they are linearly dependent on $B z$.

The $\varepsilon$ parameter of Akasofu is also a widely used function (Perreault and Akasofu, 1978; Akasofu, 1979). It is expressed by $\varepsilon=V B^{2} \sin ^{4}(\theta / 2) l_{0}^{2}$, where $l_{0}$ is $7 R_{E}, B$ is the magnitude of the IMF, and $\theta$ is the IMF clock angle defined as

$$
\begin{aligned}
& \theta=\tan ^{-1}(|B y / B z|) \quad \text { for } B z>0 \\
& \theta=180^{\circ}-\tan ^{-1}(|B y / B z|) \quad \text { for } \quad B z<0 \text {. }
\end{aligned}
$$

Thus the IMF dependence of $\varepsilon$ is expressed by $B^{2} \sin ^{4}(\theta / 2)$. Another well-known function is the general

Copy right(c) The Society of Geomagnetism and Earth, Planetary and Space Sciences (SGEPSS); The Seismological Society of Japan; The Volcanological Society of Japan; The Geodetic Society of Japan; The Japanese Society for Planetary Sciences; TERRAPUB. formula of Vasyliunas et al. (1982) derived from dimensional analysis on the magnetohydrodynamic (MHD) flow. The IMF dependence of the Vasyliunas et al. equation is expressed by a function of $M_{A} \cdot f(\theta)$, where $M_{A}$ is the Alfven Mach number, $M_{A}=V D^{1 / 2} / B_{T}, D$ is the solar wind density, $B_{T}$ is the magnetic field perpendicular to the Sun-Earth line, $B_{T}=\left(B x^{2}+B y^{2}\right)^{1 / 2}, f(\theta)$ is a function of $\theta$, and $f(\theta)$ is usually expressed by a function of $\sin (\theta / 2)$. Hence the above two coupling functions have a feature that the IMF dependence is expressed by the combination of $B$ (or $B_{T}$ ) and $\sin (\theta / 2)$.

The purpose of the present paper is to check the validity of $\varepsilon$ and of the Vasyliunas et al. formula by comparing dependence on $B z$ and dependence on $B$ (or $B_{T}$ ) and $\sin (\theta / 2$ ) using hourly values of solar wind parameters and of the $A L$ index, which is a measure of the intensity of substorms and also considered to be a measure of the solar wind energy input rate. We perform the regression analysis in a power law form and show that $B z$ is better than the combination of $B$ (or $\left.B_{T}\right)$ and $\sin (\theta / 2)$ in terms of correlation coefficients and of consistency in exponents.

\section{Data and Procedure of Analysis \\ 2.1 Data}

We use hourly values of the $A L$ index for the period from 1966 through 1987, except for 1976 and 1977 due to lack of the index. The hourly values of solar wind parameters are used for the same period. Thirty-minute delay of the $A L$ index with respect to the solar wind parameters is introduced by using averages of two consecutive $A L$ values. Hourly intervals for which all of the IMF, the solar wind velocity, the density, and the $A L$ index are available are used for the present analysis. In the next section we present the 


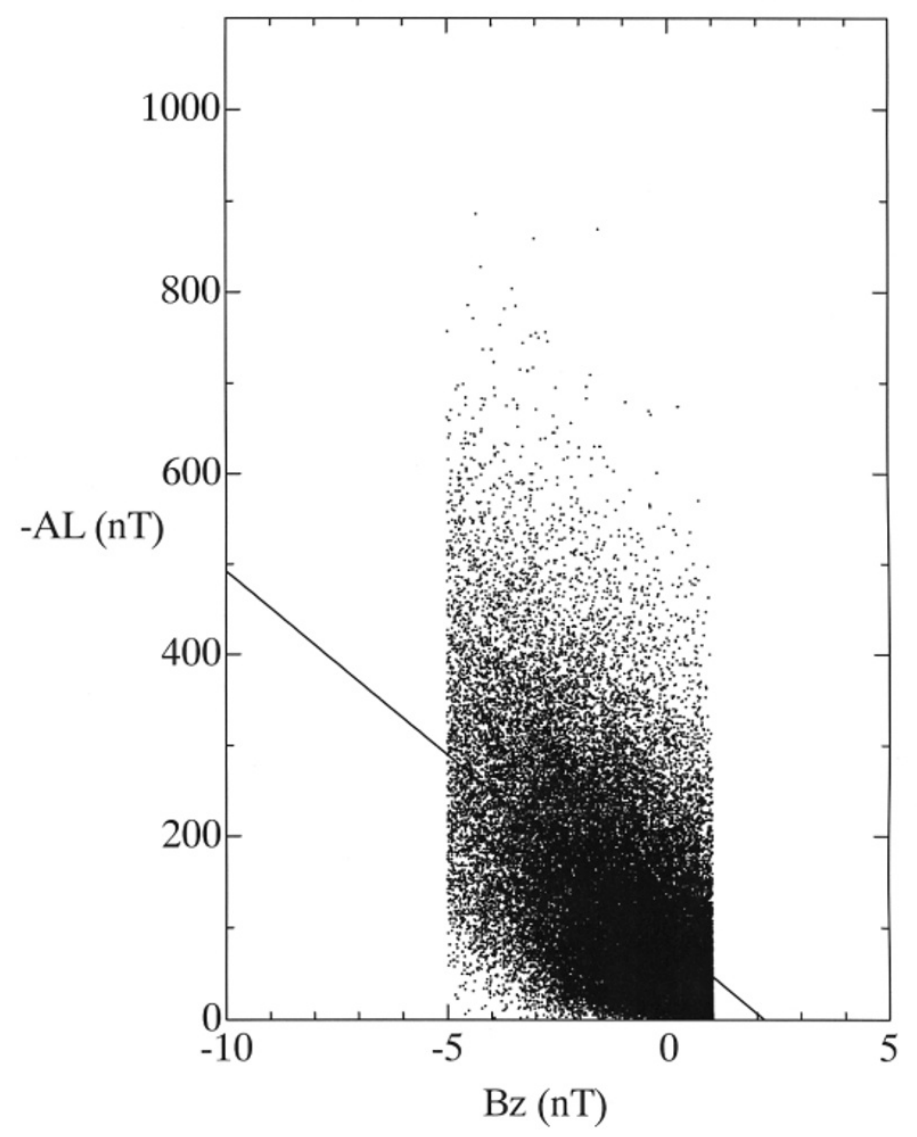

Fig. 1. Scatter plot of $A L$ versus $B z$ for the data of $-5 \leq B z<1 \mathrm{nT}$ and $V<600 \mathrm{~km} / \mathrm{s}$. The solid line gives the regression line between $A L$ and $B z$.

results for the data of the intervals where $-5 \leq B z<1$ nT and $V<600 \mathrm{~km} / \mathrm{s}$. This condition for solar wind parameters is imposed to avoid extreme situations of the solar wind. Wider range of negative $B z$ than that of positive $B z$ is chosen to avoid small values of $|A L|$, because the physical meaning of them is unclear (Allen and Kroehl, 1975; Kamide and Akasofu, 1983). The IMF components are expressed in the geocentric solar magnetospheric (GSM) coordinate system.

\subsection{Procedure of analysis}

Figure 1 shows a scatter plot of $A L$ versus $B z$ for the data of $-5 \leq B z<1 \mathrm{nT}$ and $V<600 \mathrm{~km} / \mathrm{s}$. As can be seen in this figure, the $A L$ index develops to large negative values as $B z$ becomes negative. As $B z$ approaches zero from the negative side, $A L$ becomes small negative values. This tendency does not cease at $B z=0$ and continues to some positive values of $B z$. To take account of this behavior, we performed the linear regression analysis of $A L$ on $B z$, and obtained the regression line, $A L=40.5 B z-$ 87.4 , with a correlation coefficient of 0.53 . From these regression coefficients, we obtain $B z_{0}=2.2 \mathrm{nT}$ as the value of the point at which the regression line crosses the abscissa. Below we use $B z^{\prime}=B z_{0}-B z$ instead of $B z$.

We performed the regression analysis in the form of $Y=A_{0} X_{1}^{\alpha 1}$ for each of the following parameters: $B z^{\prime}$, $B, B_{T}, \sin (\theta / 2), V, M_{A}$, and $M_{A}^{\prime}=V D^{1 / 2} / B$, the Alfven Mach number defined in terms of $B$ insted of $B_{T}$. We did this analysis by transforming $Y=A_{0} X_{1}^{\alpha 1}$ into $\log (Y)=\log \left(A_{0}\right)+\alpha_{1} \log \left(X_{1}\right)$ and by applying the linear regression analysis to this equation. We also performed the regression analysis in the form of $Y=$ $A_{0} X_{1}^{\alpha 1} X_{2}^{\alpha 2}$ for the following two-paramerer combinations: $\left(B z^{\prime}, V\right),(B, \sin (\theta / 2)),\left(B_{T}, \sin (\theta / 2)\right),\left(M_{A}, \sin (\theta / 2)\right)$, and $\left(M_{A}^{\prime}, \sin (\theta / 2)\right)$. Furthermore we did the regression analysis in the form of $Y=A_{0} X_{1}^{\alpha 1} X_{2}^{\alpha 2} X_{3}^{\alpha 3}$ for the following three-parameter combinations: $\left(B z^{\prime}, V, D\right)$, $(B, \sin (\theta / 2), V)$, and $\left(B_{T}, \sin (\theta / 2), V\right)$. We will compare the exponents and the correlation coefficients in the next section.

\section{Results}

Table 1 shows the exponents and correlation coefficients obtained by the power law fit analysis for various kinds of solar wind parameters and of their combinations. First, we compare the correlation coefficients among $B z^{\prime},(B, \sin (\theta / 2))$, and $\left(B_{T}, \sin (\theta / 2)\right)$. We notice that $B z^{\prime}$ gives the best correlation coefficient among above three quantities. This feature remains valid when we include $V$ : the combination $\left(B z^{\prime}, V\right)$ gives the highest correlation coefficient among $\left(B z^{\prime}, V\right),(B, \sin (\theta / 2), V)$, and $\left(B_{T}, \sin (\theta / 2), V\right)$. Thus we can consider that $B z^{\prime}$ is the best parameter among $B z^{\prime},(B, \sin (\theta / 2))$, and $\left(B_{T}, \sin (\theta / 2)\right)$ in terms of correlation coefficients.

We also notice the following points from Table 1:

1) The highest correlation coefficient in Table 1 is ob- 
Table 1. Exponents and correlation coefficients (c.c.) for various kinds of solar wind parameters and of their combinations obtained by the regression analysis. In this table, $\left(B_{T}, \sin (\theta / 2), V\right)$, for example, represents the case of the regression equation of log $(-A L)=$ const. $+\alpha_{1} \log \left(B_{T}\right)+\alpha_{2} \log (\sin (\theta / 2))+\alpha_{3} \log (V)$. Errors are the standard errors. The data used for every case of solar wind parameters and of their combinations are the same; the number of data points is 47,740 .

\begin{tabular}{lrccc}
\hline \multirow{2}{*}{ parameters } & \multicolumn{3}{c}{ exponents } & \multicolumn{1}{c}{ c.c. $^{*}$} \\
\cline { 2 - 4 } & $1.107 \pm 0.009$ & $\alpha_{2}$ & 0.508 \\
$B z^{\prime}$ & $1.021 \pm 0.011$ & & & 0.404 \\
$B$ & $0.492 \pm 0.006$ & & 0.350 \\
$B_{T}$ & $1.050 \pm 0.014$ & & 0.317 \\
$\sin (\theta / 2)$ & $2.272 \pm 0.022$ & & & 0.423 \\
$V$ & $-0.317 \pm 0.006$ & & & 0.232 \\
$M_{A}$ & $-0.417 \pm 0.010$ & & & 0.183 \\
$M_{A}^{\prime}$ & $1.118 \pm 0.007$ & $2.303 \pm 0.018$ & & 0.665 \\
$\left(B z^{\prime}, V\right)$ & $0.929 \pm 0.010$ & $0.884 \pm 0.013$ & & 0.483 \\
$(B, \sin (\theta / 2))$ & $0.398 \pm 0.006$ & $0.783 \pm 0.014$ & & 0.417 \\
$\left(B_{T}, \sin (\theta / 2)\right)$ & $-0.219 \pm 0.006$ & $0.912 \pm 0.015$ & & 0.353 \\
$\left(M_{A}, \sin (\theta / 2)\right)$ & $-0.351 \pm 0.010$ & $1.002 \pm 0.014$ & & 0.352 \\
$\left(M_{A}^{\prime}, \sin (\theta / 2)\right)$ & $1.088 \pm 0.007$ & $2.680 \pm 0.020$ & $0.247 \pm 0.005$ & 0.682 \\
$\left(B z^{\prime}, V, D\right)$ & $0.754 \pm 0.009$ & $0.921 \pm 0.012$ & $1.985 \pm 0.020$ & 0.604 \\
$(B, \sin (\theta / 2), V)$ & $0.400 \pm 0.005$ & $0.788 \pm 0.013$ & $2.284 \pm 0.020$ & 0.596 \\
$\left(B_{T}, \sin (\theta / 2), V\right)$ & & & & \\
\hline
\end{tabular}

${ }^{*}$ Correlation coefficient.

tained for the combination of $\left(B z^{\prime}, V, D\right)$.

2) The correlation coefficients for $M_{A}, \quad M_{A}^{\prime}$, $\left(M_{A}, \sin (\theta / 2)\right)$, and $\left(M_{A}^{\prime}, \sin (\theta / 2)\right)$ are very small compared with those for $B z^{\prime},(B, \sin (\theta / 2))$, and $\left(B_{T}, \sin (\theta / 2)\right)$.

3) The exponent for $B z^{\prime}$ is approximately equal to unity. This feature is seen in common in all cases of $B z^{\prime}$, $\left(B z^{\prime}, V\right)$, and $\left(B z^{\prime}, V, D\right)$.

4) The exponent for $B$ is roughly equal to unity in the cases of $B,(B, \sin (\theta / 2))$, and $(B, \sin (\theta / 2), V)$. It is never equal to two, as expected from $\varepsilon$.

5) The exponent for $B_{T}$ is roughly equal to 0.5 in the cases of $B_{T},\left(B_{T}, \sin (\theta / 2)\right)$, and $\left(B_{T}, \sin (\theta / 2), V\right)$.

6) The exponent for $\sin (\theta / 2)$ is roughly equal to unity in all cases including $\sin (\theta / 2): \sin (\theta / 2),(B, \sin (\theta / 2))$ $\left(B_{T}, \sin (\theta / 2)\right), \quad\left(M_{A}, \sin (\theta / 2)\right), \quad\left(M_{A}^{\prime}, \sin (\theta / 2)\right)$, $(B, \sin (\theta / 2), V)$, and $\left(B_{T}, \sin (\theta / 2), V\right)$. It is never equal to four, as expected from $\varepsilon$.

7) The exponent for $V$ is roughly equal to two, as in $B s V^{2}$ obtained by Murayama and Hakamada (1975), not to unity, as expected from $\varepsilon$. This feature is seen in common in all cases including $V: V,\left(B z^{\prime}, V\right)$, $\left(B z^{\prime}, V, D\right),(B, \sin (\theta / 2), V)$, and $\left(B_{T}, \sin (\theta / 2), V\right)$.

\section{Discussion}

\subsection{Influence of the conditions of data selection on the results}

We obtained the results in the preceding section under the condition of $-5 \leq B z<1 \mathrm{nT}$ and $V<600 \mathrm{~km} / \mathrm{s}$. In this subsection we discuss the influence of the conditions of data selection on the results.

We performed the same analysis as the one in the preceding section by changing the conditions for the solar wind parameters. As for the condition on $B z$ we investigated the following seventeen ranges: $[-3,1]$ (which means $-3 \leq$ $B z<1 \mathrm{nT}$; we use the same format below), [-4, 1], $[-5,1],[-6,1],[, 1],[-4,0],[-5,0],[-6,0],[-7,0]$, $[, 0],[-5,-1],[-6,-1],[-7,-1],[,-1],[-5,-2]$, $[-6,-2]$, and [,-2$]$. As for $V$, we examined the following three ranges: $V<600 \mathrm{~km} / \mathrm{s}, V \geq 600 \mathrm{~km} / \mathrm{s}$, and all $V$. We analysed all cases of any combinations of the above $B z$ and $V$ ranges.

Table 2 shows the examples of this analysis: results for $B z^{\prime}$, for $\sin (\theta / 2)$, and for the combination of $\left(B_{T}, \sin (\theta / 2)\right)$ for various ranges of $B z$ and for the range of $V<600 \mathrm{~km} / \mathrm{s}$. From this table we notice that in some $B z$ ranges the combination of $\left(B_{T}, \sin (\theta / 2)\right)$ shows slightly better correlation coefficients than $B z^{\prime}$. However, the exponents for $B_{T}$ and for $\sin (\theta / 2)$ show larger variability for different ranges of $B z$ than those for $B z^{\prime}$ : the exponents for $B z^{\prime}$ range from 1.09 to 1.30 , those for $B_{T}$ from 0.31 to 0.91 , and those for $\sin (\theta / 2)$ from 0.63 to 4.40 (from 0.37 to 3.04 for single parameter case). Thus the exponents for $B z$ show higher consistency than those for $B_{T}$ and for $\sin (\theta / 2)$ in the combination of $\left(B_{T}, \sin (\theta / 2)\right)$ for the change in the range of $B z$.

The above feature was more generally confirmed as follows: The combinations of $\left(B z^{\prime}, V\right)$ and of $\left(B z^{\prime}, V, D\right)$ always show good correlation coefficients, and $\left(B z^{\prime}, V, D\right)$, in paticular, almost always gives the best correlation coefficient among the solar wind parameters and their combinations listed in Table 1 for every range of $B z$. The combinations of $\left(B_{T}, \sin (\theta / 2), V\right)$ and of $(B, \sin (\theta / 2), V)$ usually show good correlation coefficients, and $\left(B_{T}, \sin (\theta / 2), V\right)$, 
Table 2. Exponents and correlation coefficients (c.c.) for $B z^{\prime}$, for $\sin (\theta / 2)$, and for the combination of $B_{T}$ and $\sin (\theta / 2)$ for various ranges of $B z$ and for $V<600 \mathrm{~km} / \mathrm{s}$.

\begin{tabular}{|c|c|c|c|c|c|c|c|c|c|}
\hline \multirow{2}{*}{$B z$ range } & \multirow{2}{*}{$\begin{array}{l}\text { Number of } \\
\text { data points }\end{array}$} & \multirow{2}{*}{$B z_{0}$} & \multicolumn{2}{|c|}{$B z^{\prime}$} & \multicolumn{2}{|c|}{$\sin (\theta / 2)$} & \multicolumn{3}{|c|}{$\left(B_{T}, \sin (\theta / 2)\right)$} \\
\hline & & & $\alpha$ & c.c. ${ }^{*}$ & $\alpha$ & c.c.* & $\alpha_{1}$ & $\alpha_{2}$ & c.c. ${ }^{*}$ \\
\hline$[-3,1]$ & 40818 & 2.5 & $1.13 \pm 0.01$ & 0.405 & $0.80 \pm 0.02$ & 0.257 & $0.31 \pm 0.01$ & $0.63 \pm 0.02$ & 0.340 \\
\hline$[-4,1]$ & 45056 & 2.3 & $1.11 \pm 0.01$ & 0.467 & $0.95 \pm 0.02$ & 0.293 & $0.36 \pm 0.01$ & $0.73 \pm 0.02$ & 0.384 \\
\hline$[-5,1]$ & 47740 & 2.2 & $1.11 \pm 0.01$ & 0.508 & $1.05 \pm 0.01$ & 0.317 & $0.40 \pm 0.01$ & $0.78 \pm 0.01$ & 0.417 \\
\hline$[-6,1]$ & 49407 & 2.1 & $1.09 \pm 0.01$ & 0.535 & $1.12 \pm 0.01$ & 0.332 & $0.43 \pm 0.01$ & $0.82 \pm 0.01$ & 0.441 \\
\hline$[\quad, 1]$ & 52270 & 2.3 & $1.17 \pm 0.01$ & 0.596 & $1.28 \pm 0.01$ & 0.362 & $0.50 \pm 0.01$ & $0.85 \pm 0.01$ & 0.497 \\
\hline$[-4,0]$ & 32971 & 1.8 & $1.20 \pm 0.01$ & 0.423 & $1.74 \pm 0.05$ & 0.196 & $0.58 \pm 0.01$ & $2.96 \pm 0.05$ & 0.438 \\
\hline$[-5, \quad 0]$ & 35655 & 1.7 & $1.18 \pm 0.01$ & 0.473 & $2.15 \pm 0.05$ & 0.243 & $0.61 \pm 0.01$ & $3.13 \pm 0.04$ & 0.483 \\
\hline$[-6,0]$ & 37322 & 1.7 & $1.18 \pm 0.01$ & 0.505 & $2.42 \pm 0.04$ & 0.273 & $0.62 \pm 0.01$ & $3.22 \pm 0.04$ & 0.512 \\
\hline$[-7,0]$ & 38330 & 1.7 & $1.19 \pm 0.01$ & 0.528 & $2.61 \pm 0.04$ & 0.293 & $0.63 \pm 0.01$ & $3.28 \pm 0.04$ & 0.533 \\
\hline$\left[\begin{array}{ll}, & 0\end{array}\right]$ & 40185 & 2.1 & $1.27 \pm 0.01$ & 0.575 & $3.04 \pm 0.04$ & 0.336 & $0.65 \pm 0.01$ & $3.37 \pm 0.04$ & 0.580 \\
\hline$[-5,-1]$ & 23383 & 1.4 & $1.18 \pm 0.02$ & 0.398 & $0.93 \pm 0.07$ & 0.092 & $0.82 \pm 0.01$ & $3.88 \pm 0.08$ & 0.412 \\
\hline$[-6,-1]$ & 25050 & 1.5 & $1.20 \pm 0.02$ & 0.440 & $1.29 \pm 0.06$ & 0.126 & $0.82 \pm 0.01$ & $3.90 \pm 0.07$ & 0.452 \\
\hline$[-7,-1]$ & 26058 & 1.5 & $1.20 \pm 0.01$ & 0.470 & $1.54 \pm 0.06$ & 0.149 & $0.83 \pm 0.01$ & $3.93 \pm 0.06$ & 0.480 \\
\hline$[\quad,-1]$ & 27913 & 2.3 & $1.30 \pm 0.01$ & 0.534 & $2.17 \pm 0.06$ & 0.205 & $0.81 \pm 0.01$ & $3.87 \pm 0.06$ & 0.545 \\
\hline$[-5,-2]$ & 13657 & 1.2 & $1.20 \pm 0.03$ & 0.314 & $0.37 \pm 0.09$ & 0.035 & $0.91 \pm 0.02$ & $4.40 \pm 0.13$ & 0.323 \\
\hline$[-6,-2]$ & 15324 & 1.4 & $1.21 \pm 0.03$ & 0.365 & $0.70 \pm 0.09$ & 0.065 & $0.89 \pm 0.02$ & $4.29 \pm 0.11$ & 0.372 \\
\hline$[\quad,-2]$ & 18187 & 2.8 & $1.30 \pm 0.02$ & 0.491 & $1.70 \pm 0.08$ & 0.151 & $0.83 \pm 0.01$ & $4.00 \pm 0.08$ & 0.500 \\
\hline
\end{tabular}

in paticular, sometimes shows nearly the same level of correlation coefficient as $\left(B z^{\prime}, V, D\right)$. However, the exponents for $B_{T}$ and for $\sin (\theta / 2)$ in the combination of $\left(B_{T}, \sin (\theta / 2), V\right)$ show larger variability than those for $B z^{\prime}$. Also the exponents for $B$ and for $\sin (\theta / 2)$ in the cases of $B,(B, \sin (\theta / 2))$, and $(B, \sin (\theta / 2), V)$ show larger variability than those for $B z^{\prime}$. Hence we have reached the same conclusion, again, as in the preceding section that $B z$ generally gives high correlation coefficients and shows higher consistency in exponents than the combination of $B_{T}$ (or $B)$ and $\sin (\theta / 2)$ from the analysis for the various kinds of conditions on the data selection.

Here we would like to point out one fact. There exist some cases where the exponents for $\sin (\theta / 2)$ are roughly four (e.g., Table 2), but even in those cases the exponents for $B_{T}$ (or $B$ ) are not two, as expected from the $\varepsilon$ parameter. Thus we never obtained the $B_{T}^{2} \sin ^{4}(\theta / 2)$ (or $B^{2} \sin ^{4}(\theta / 2)$ ) dependence from the present analysis.

In the above, we imposed restrictions on the basis of the values of $B z$ and made logarithmic analyses mainly in the negative range of $B z$. This methodology itself might work in favor of $B z$ and produce the result of a better performance of $B z$. In order to check this possibility, we imposed restrictions on the basis of the values of $\theta$ and chose the ranges so that each of the ranges is symmetric with respect to $90^{\circ}: 90-10 p \leq \theta<90+10 p^{\circ},(p=$ $1,2, \cdots, 9)$. For each range of $\theta$, we performed the linear regression analysis of $A L$ on $V^{l} B^{m} \sin ^{n}(\theta / 2),(l=0,1$; $m=0,2 ; n=1,2,4)$, on $V^{l} B_{T}^{m} \sin ^{n}(\theta / 2),(l=0,1$; $m=0,2 ; n=1,2,4)$, and on $V^{l} B z c,(l=0,2)$, where $B z c$ is defined as

$$
\begin{array}{ll}
B z c=2.2-B z & \text { for } \quad B z<2.2 \mathrm{nT} \\
B z c=0 & \text { for } \quad B z \geq 2.2 \mathrm{nT} .
\end{array}
$$

(a)
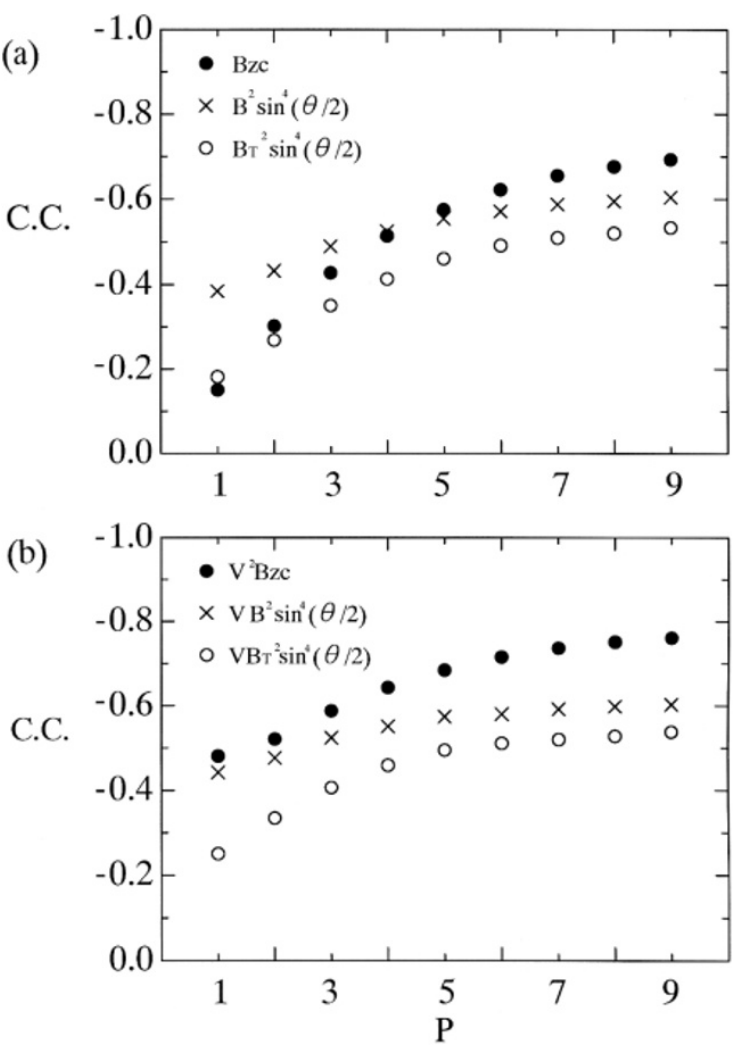

Fig. 2. (a) Correlation coefficients (c.c.) of $A L$ with $B z c$ (solid circles), with $B^{2} \sin ^{4}(\theta / 2)$ (crosses), and with $B_{T}^{2} \sin ^{4}(\theta / 2)$ (open circles) for the ranges of $90-10 p \leq \theta<90+10 p^{\circ},(p=1,2, \cdots, 9)$ and for the range of $V<600 \mathrm{~km} / \mathrm{s}$. Values of the abscissa are the values of $p$. (b) Correlation coefficients (c.c.) of $A L$ with $V^{2} B z c$ (solid circles), with $V B^{2} \sin ^{4}(\theta / 2)$ (crosses), and with $V B_{T}^{2} \sin ^{4}(\theta / 2)$ (open circles) for the ranges of $90-10 p \leq \theta<90+10 p^{\circ},(p=1,2, \cdots, 9)$ and for the range of $V<600 \mathrm{~km} / \mathrm{s}$. 
Table 3. Linear correlation coefficients (c.c.) between solar wind parameters for various ranges of $B z$ and for $V<600 \mathrm{~km} / \mathrm{s}$. In this table, $\langle B z$, $\sin (\theta / 2)\rangle$,

for example, represents the case for the linear regression analysis between $B z$ and $\sin (\theta / 2)$. The same data as those in Table 2 are used for each range of $B z$.

\begin{tabular}{|c|c|c|c|c|c|c|c|c|c|c|c|}
\hline$B z$ range & $\begin{array}{l}\text { Number of } \\
\text { data points }\end{array}$ & $\langle B z, \sin (\theta / 2)\rangle$ & $\langle B z, B\rangle$ & $\left\langle B z, B_{T}\right\rangle$ & $\langle B z, V\rangle$ & $\langle\sin (\theta / 2), B\rangle$ & $\left\langle\sin (\theta / 2), B_{T}\right\rangle$ & $\langle\sin (\theta / 2), V\rangle$ & $\left\langle B, B_{T}\right\rangle$ & $\langle B, V\rangle$ & $\left\langle B_{T}, V\right\rangle$ \\
\hline$[-3,1]$ & 40818 & -0.768 & -0.136 & -0.190 & 0.015 & 0.010 & 0.027 & -0.005 & 0.763 & 0.162 & -0.007 \\
\hline$[-4,1]$ & 45056 & -0.758 & -0.212 & -0.282 & 0.014 & 0.039 & 0.065 & -0.005 & 0.775 & 0.156 & -0.008 \\
\hline$[-5,1]$ & 47740 & -0.746 & -0.274 & -0.359 & 0.012 & 0.068 & 0.103 & -0.005 & 0.785 & 0.154 & -0.005 \\
\hline$[-6, \quad 1]$ & 49407 & -0.734 & -0.328 & -0.419 & 0.013 & 0.094 & 0.134 & -0.007 & 0.794 & 0.152 & -0.005 \\
\hline$[\quad, 1]$ & 52270 & -0.651 & -0.550 & -0.630 & -0.008 & 0.176 & 0.218 & -0.005 & 0.843 & 0.149 & 0.014 \\
\hline$[-4, \quad 0]$ & 32971 & -0.629 & -0.246 & -0.324 & 0.011 & -0.248 & -0.370 & 0.006 & 0.777 & 0.152 & -0.008 \\
\hline$[-5,0]$ & 35655 & -0.637 & -0.308 & -0.402 & 0.008 & -0.200 & -0.294 & 0.004 & 0.789 & 0.151 & -0.005 \\
\hline$[-6, \quad 0]$ & 37322 & -0.638 & -0.364 & -0.464 & 0.009 & -0.155 & -0.233 & 0.002 & 0.799 & 0.149 & -0.004 \\
\hline$[-7,0]$ & 38330 & -0.636 & -0.410 & -0.511 & 0.009 & -0.121 & -0.187 & 0.001 & 0.810 & 0.147 & -0.002 \\
\hline$\left[\begin{array}{ll}, & 0\end{array}\right]$ & 40185 & -0.583 & -0.599 & -0.680 & -0.017 & -0.003 & -0.044 & 0.003 & 0.853 & 0.149 & 0.020 \\
\hline$[-5,-1]$ & 23383 & -0.410 & -0.295 & -0.389 & 0.001 & -0.417 & -0.576 & 0.019 & 0.789 & 0.147 & -0.001 \\
\hline$[-6,-1]$ & 25050 & -0.432 & -0.358 & -0.461 & 0.003 & -0.367 & -0.502 & 0.015 & 0.800 & 0.147 & 0.001 \\
\hline$[-7,-1]$ & 26058 & -0.443 & -0.410 & -0.516 & 0.002 & -0.326 & -0.442 & 0.013 & 0.812 & 0.145 & 0.004 \\
\hline$[\quad,-1]$ & 27913 & -0.429 & -0.629 & -0.711 & -0.031 & -0.163 & -0.231 & 0.014 & 0.860 & 0.152 & 0.033 \\
\hline$[-5,-2]$ & 13657 & -0.252 & -0.256 & -0.342 & -0.005 & -0.558 & -0.738 & 0.020 & 0.794 & 0.149 & 0.005 \\
\hline$[-6,-2]$ & 15324 & -0.288 & -0.327 & -0.426 & -0.001 & -0.510 & -0.664 & 0.014 & 0.803 & 0.151 & 0.008 \\
\hline$[\quad,-2]$ & 18187 & -0.327 & -0.652 & -0.733 & -0.048 & -0.263 & -0.324 & 0.009 & 0.868 & 0.165 & 0.052 \\
\hline
\end{tabular}


We did this analysis for the following three ranges of $V$ : $V<600 \mathrm{~km} / \mathrm{s}, V \geq 600 \mathrm{~km} / \mathrm{s}$, and all $V$.

Figures 2(a) and 2(b) show the correlation coefficients between $A L$ and various parameters for different ranges of $\theta$ and for $V<600 \mathrm{~km} / \mathrm{s}$. Both figures clearly show the general superiority of $B z$ over $B^{2} \sin ^{4}(\theta / 2)$ and over $B_{T}^{2} \sin ^{4}(\theta / 2)$. Other results (not shown) also support the conclusion that $B z$ is better than the combination of $B$ (or $\left.B_{T}\right)$ and $\sin (\theta / 2)$.

\subsection{Influence of solar wind parameters other than $B z$ and $V$ on the results}

In the Results section we mainly analysed the effects of $B z$ and of $V$. There exist, however, other solar wind parameters which are known to influence the value of $A L$. Those are the solar wind density (e.g., Maezawa, 1978), the IMF $B y$ component, and the dipole tilt angle $(\chi)$ (Aoki, 1977; Hakamada et al., 1980; Murayama et al., 1980). The dipole tilt angle is defined as the geomagnetic latitude of the subsolar point. In this subsection we discuss the influence of these parameters on the results.

First, we consider the effect of the solar wind density. As can be seen in $\left(B z^{\prime}, V, D\right)$ in Table $1, D$ really improves the correlation coefficient compared with the correlation coefficient of $\left(B z^{\prime}, V\right)$, but this improvement is very small. The exponent for the density is also very small. Thus we can expect that the density would not affect the results of the preceding section.

Second, we discuss the effects of $B y$ and of $\chi$. The main effect of $B y$ is almost linear for a given sign of $\chi$, and the gradient of the regression line reverses its sign for different signs of $\chi$ (Hakamada et al., 1980). Below we call this phenomenon the $B y-\chi$ effect. This effect also would have little influence on our results, because this would be averaged out by using means for more than one year because of the annual periodicity of the value of $\chi$. The dipole tilt angle also has an effect of maximizing geomagnetic activity at $\chi=0^{\circ}$. This effect would also be leveled off by averaging data for more than one year. Thus we can expect that $B y$ and $\chi$ do not change the results in the preceding section.

On the other hand, the existence of the $B y-\chi$ effect shows a great difficulty to the idea that the IMF dependence of the $A L$ index can be described by the combination of $B$ (or $B_{T}$ ) and $\sin (\theta / 2)$. The reason is as follows: Each of the following three quantities, $B, B_{T}$, and $\sin (\theta / 2)$, is symmetric with respect to the sign of $B y$ (i.e., concerning the influence of $B y$, each quantity is determined by the absolute value of $B y$ and has no relation to the sign of $B y$ ), while obviously the $B y-\chi$ effect is not symmetric with respect to it. Thus the $B y-\chi$ effect cannot be described by the combination of $B$ (or $B_{T}$ ) and $\sin (\theta / 2)$.

\subsection{Influence of intercorrelations among solar wind parameters}

It is well-known that solar wind parameters show intercorrelations among themselves. In this subsection we discuss the influence of these correlations on the results.

We performed the linear regression analysis for any combinations of the following solar wind paramers: $B z$, $\sin (\theta / 2), B, B_{T}$, and $V$. Table 3 shows the correlation coefficients for various ranges of $B z$ and for $V<600 \mathrm{~km} / \mathrm{s}$. Correlation coefficients for the ranges of $V \geq 600 \mathrm{~km} / \mathrm{s}$ and for all $V$ are almost the same as those in Table 3 (not shown).

From this table we notice that the correlation coefficients for the combinations containing $V$ are generally very small: correlation coefficients between $V$ and $B$ are slightly positive values, and correlation coefficients for other combinations are almost zero. This fact guarantees that we can discuss dependence on the IMF without considering the existence of dependence on $V$ in the coupling function.

\subsection{Remaining problems}

In the present paper, we have used the $A L$ index as a measure of the rate of the solar wind energy input into the magnetosphere. Actually the $A L$ index reflects the intensity of the westward electrojets flowing in the ionosphere of the auroral zone. The energy transferred through the solar wind-magnetosphere interaction is distributed to various regions in the magnetosphere and produces a lot of phenomena other than the auroral electrojets, such as ring current intensification and plasmoid release. So to investigate the accurate functional form of the soalr wind-magnetosphere coupling we should compare the total energy with solar wind parameters. These comparisons, however, are not an easy task because of some uncertainties such as lack of accurate knowledge of decay function of the ring current and lack of the precise method of measuring energy carried by plasmoids. In this sense the present study is considered as a first step toward more appropriate investigations. We are now investigating the solar wind parameter dependence of the ring current injection rate and preparing a paper.

\section{Conclusions}

We have examined the IMF dependence of the $A L$ index by comparing the influence of $B z$ and that of the combination of $B$ (or $B_{T}$ ) and $\sin (\theta / 2)$. The regression analysis in a power law form shows that $B z$ generally gives high correlation coefficients and shows higher consistency in exponents. Higher consistency of $B z$ can be seen in the fact that the exponent for $B z^{\prime}$ shows smaller variability for the change in the range of $B z$ (Table 2). This fact implies that the IMF dependence of the $A L$ index is described better by $B z$ than by the combination of $B$ (or $B_{T}$ ) and $\sin (\theta / 2)$, and that the $\varepsilon$ parameter of Akasofu and the general formula of Vasyliunas et al. (1982) for the energy coupling function are less appropriate than a function of $B z$.

Acknowledgments. Data of the interplanetary magnetic field and the solar wind were provided by Dr. J. H. King through the WDCA for Rockets and Satellites in GSFC/NASA. Data of the AL index were provided through the World Data Center C2 for Geomagnetism at Kyoto University and the National Geophysical Data Center.

\section{References}

Akasofu, S.-I., Interplanetary energy flux associated with magnetospheric substorms, Planet. Space Sci., 27, 425-431, 1979.

Allen, J. H. and H. W. Kroehl, Spatial and temporal distributions of magnetic effects of auroral electrojets as derived from AE indices, J. Geophys. Res., 80, 3667-3677, 1975.

Aoki, T., Influence of the dipole tilt angle on the development of auroral electrojets, J. Geomag. Geoelectr., 29, 441-453, 1977.

Baker, D. N., Statistical analyses in the study of solar wind-magnetosphere coupling, in Solar Wind-Magnetosphere Coupling, edited by Y. Kamide and J. A. Slavin, pp. 17-38, Terrapub/Reidel, Tokyo, 1986. 
Hakamada, K., T. Aoki, and T. Murayama, Influence of the east-west component of the interplanetary magnetic field on the intensity of the auroral electrojet, Planet. Space Sci., 28, 29-39, 1980.

Kamide, Y. and S.-I. Akasofu, Notes on the auroral electrojet indices, Rev. Geophys. Space Phys., 21, 1647-1656, 1983.

Koskinen, H. E. J. and E. I. Tanskanen, Magnetospheric energy budget and the epsilon parameter, J. Geophys. Res., 107(A11), 1415 doi:10.1029/2002JA009283, 2002.

Maezawa, K., Statistical study of the dependence of geomagnetic activity on solar wind parameters, in Quantitative Modeling of Magnetospheric Processes, Geophys. Monogr. Ser., vol. 21, edited by W. P. Olson, pp. 436-447, AGU, Washington, D.C., 1979.

Murayama, T. and K. Hakamada, Effects of solar wind parameters on the development of magnetospheric substorms, Planet. Space Sci., 23, 7591,1975
Murayama, T., T. Aoki, H. Nakai, and K. Hakamada, Empirical formula to relate the auroral electrojet intensity with interplanetary parameters, Planet. Space Sci., 28, 803-813, 1980.

Perreault, P. and S.-I. Akasofu, A study of geomagnetic storms, Geophys. J. R. Astron. Soc., 54, 547-573, 1978.

Rostoker, G., H. L. Lam, and W. D. Hume, Response time of the magnetosphere to the interplanetary electric field, Can. J. Phys., 50, 544-547, 1972.

Vasyliunas, V. M., J. R. Kan, G. L. Siscoe, and S.-I. Akasofu, Scaling relations governing magnetospheric energy transfer, Planet. Space Sci., 30, 359-365, 1982.

T. Aoki (e-mail: aoki317@ruby.ocn.ne.jp) 